\title{
Première observation de l'éponge introduite et invasive Celtodoryx ciocalyptoides (Burton, 1935) (Porifera : Coelosphaeridae) dans le port du Havre (Manche)
}

\section{First observation of the alien and invasive sponge Celtodoryx ciocalyptoides (Burton, 1935) (Porifera: Coelosphaeridae) in the port of Le Havre (English Channel)}

\author{
Aurélien Berno ${ }^{1,2}$, Chloé Dancie ${ }^{1}$, Lucas Pinsivy ${ }^{1}$, Denis Corthésy², \\ Gérard Breton ${ }^{2,3}$
}

1 Cellule de Suivi du Littoral Normand, (CSLN), 53 rue de Prony, 76600 Le Havre

2 Association Port Vivant, 42 rue Daubrée, 76620 Le Havre gerard-breton@orange.fr

36 rue des Réservoirs. 76600 Le Havre gerard-breton@orange.fr

Résumé - L'objectif de cette étude est de signaler une première observation en Manche orientale et l'introduction dans le port du Havre du spongiaire Celtodoryx ciocalyptoides (Burton, 1935), et de comparer la population havraise avec celles du Pacifique nord-ouest (première description de l'espèce) et celles implantées dans les années 1990 dans le sud de la Bretagne (France) et dans l'Oosterschelde (Pays-Bas). La petite population havraise a été observée en 2014 sur des enrochements de la berge d'un bassin de marée, entre $+1 \mathrm{~m}$ et $-4,8 \mathrm{~m}$. Les trois populations européennes (Le Havre, Bretagne-sud et Pays-Bas) aussi bien que la population du Pacifique nord-ouest se sont toutes développées dans un environnement paralique, c'est-à-dire dans une zone de transition entre milieu marin et milieu continental. Le vecteur d'introduction est vraisemblablement le trafic maritime et ne peut pas être l'importation de naissain de l'huître Crassostrea gigas (Thunberg, 1793) pour l'ostréiculture, vecteur invoqué pour les deux précédentes introductions de cette espèce en Europe, en raison de l'absence de parcs ostréicoles à proximité.

Mots-clés - Porifera, espèce invasive, espèce introduite, domaine paralique, port du Havre.

Abstract - The aim of this paper is to notice a first occurrence in the Eastern English Channel and the introduction in the port of Le Havre of the sponge Celtodoryx ciocalyptoides (Burton, 1935), and to compare the population from Le Havre with the one from the North West Pacific (first description of the species), and the two populations that settled down in the 90's in South Brittany (France) and the Oosterschelde (The Netherlands). The 
small population in the port of Le Havre has been found in 2014 at the rocky shore of a tidal dock, between $+1 \mathrm{~m}$ and $-4.8 \mathrm{~m}$. The three European populations (Le Havre, South Brittany and Oosterschelde) and the Pacific population all developed in a paralic environment, defined as a transition zone between marine and continental systems. The introduction vector in Le Havre port is likely shipping and cannot be the importation of the spat of Crassostrea gigas (Thunberg, 1793) for farming, as suspected for the two previous European introductions of this species, given the lack of oyster farms in the vicinity.

Key words - Porifera, invasive species, introduced species, paralic domain, port of Le Havre

\section{INTRODUCTION}

Les invasions biologiques sont une des principales menaces pour la biodiversité mondiale. En milieu marin, les eaux de ballast, transportées par les grands navires marchands, le biofouling ou les débris flottants sont reconnus comme des causes majeures d'introduction d'espèces. Or l'introduction d'espèces peut avoir des répercussions sur l'écosystème receveur en modifiant les réseaux trophiques (OcchipintiAmbrogi, 2007). De plus, les espèces introduites peuvent être des vecteurs de parasitisme ou de perturbations du milieu (Kuris et Culver, 1999) ou encore devenir des compétiteurs pour la nourriture et/ou l'espace (Geller, 1999 ; Jousson et al., 2000).

Les inventaires faunistiques réalisés en particulier dans le port du Havre par la Cellule de Suivi du Littoral Normand (CSLN) et l'association Port Vivant (Breton, 2005, 2014) ont conduit en 2014 à la découverte d'une population d'un spongiaire jusque-là inconnu dans le port du Havre. L'étude in situ et au laboratoire de cette éponge a montré qu'elle appartenait à l'espèce Celtodoryx ciocalyptoides (Burton, 1935). Notre travail tente de comparer la population havraise avec les populations précédemment décrites (voir le paragraphe 2.
HISTORIQUE) et leurs contextes écologiques, et de discuter le mode d'introduction de l'espèce dans le port du Havre.

\section{HISTORIQUE}

Perez et al. (2006) avaient décrit, sous le nom de Celtodoryx girardae Perez, Perrin, Carteron, Vacelet \& Boury-Esnault, 2006 une espèce de spongiaire opportuniste qu'ils pensaient nouvelle et qui avait été repérée pour la première fois en 1996 dans la ria d'Etel (Morbihan) puis, depuis 1999, dans le golfe du Morbihan (Bretagne méridionale, France) (Fig. 1). Cette même espèce de spongiaire est décrite comme invasive dans l'Oosterschelde (Pays-Bas) par van Soest et al. (2007), son origine restant inconnue. Le vecteur d'introduction invoqué est le naissain de l'huître Crassostrea gigas (Thunberg, 1793) importé pour l'ostréiculture depuis la décennie 1990 à la fois dans le golfe du Morbihan et dans l'Oosterschelde. Henkel \& Janussen (2011) montrent que Celtodoryx girardae est un synonyme plus récent de Celtodoryx ciocalyptoides (Burton, 1935). En accord avec van Soest et al. (2007), Henkel \& Janussen pensent que l'espèce a probablement été introduite depuis son aire d'origine, le Pacifique nord-ouest (mer du Japon, mer Jaune) dans l'Atlantique 


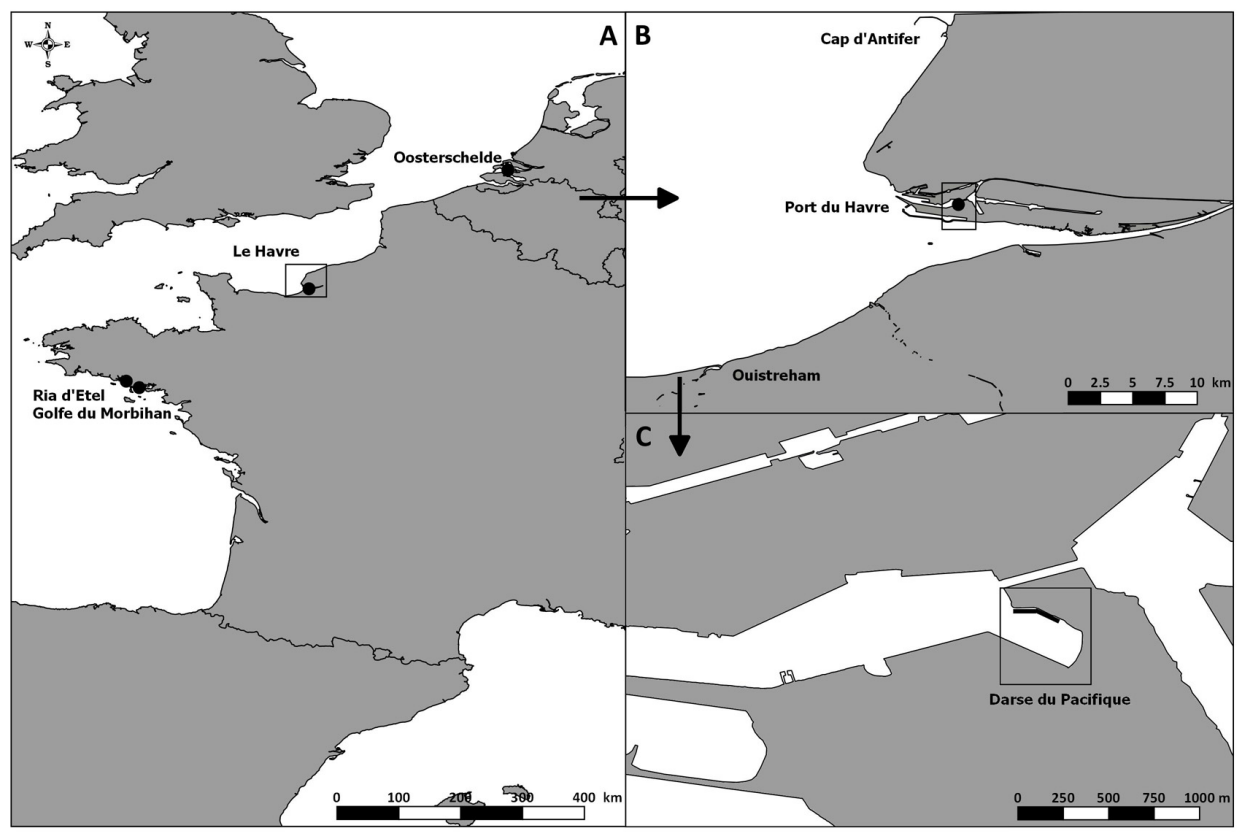

Fig. 1. A : Localisation des trois occurrences de Celtodoryx ciocalyptoides (Burton, 1935) en Europe, ria d'Etel et golfe du Morbihan, Oosterschelde, port du Havre. B : Carte générale du port du Havre, de l'estuaire de la Seine et de la partie orientale de la baie de Seine. Localisation de Ouistreham ; point noir : Darse du Pacifique. C : Carte du port du Havre. Le trait plein épais à l'intérieur du cadre carré délimite l'extension de Celtodoryx ciocalyptoides.

Fig. 1. A: Location of the three European occurrences of Celtodoryx ciocalyptoides (Burton, 1935). Ria of Etel and gulf of Morbihan, Oosterschelde, port of Le Havre. B: General map of the port of Le Havre, the Seine estuary and the eastern part of the baie de Seine. Location of Ouistreham, solid black dot: Darse du Pacifique. C: Map of the port of Le Havre. The solid black line within the square frame shows the extension of Celtodoryx ciocalyptoides.

nord-est (golfe du Morbihan, Oosterschelde en mer du Nord) par l'ostréiculture de Crassostrea gigas.

\section{MATÉRIEL ET MÉTHODES}

Les explorations à pied sont faites à basse mer de vive-eau par la CSLN $(A B, C D, L P)$ et les explorations en scaphandre autonome à marée haute ou moyenne par l'association Port Vivant (DC, GB et autres plongeurs) au niveau de l'estran de la Darse du Pacifique, Port du Havre, autour du point $49^{\circ}$
28,37' $\mathrm{N} ; 0^{\circ}$ 10,67 E (WGS 84). Les prises de vues sous-marines sont argentiques (Nikon RS) ou numériques (Nikon D 80 en caisson).

Les microscopes utilisés sont les suivants: Nachet 400 équipé d'un boîtier D 80 ou D 90 (fond clair, contraste interférentiel Nomarski) et Leitz Laborlux $\mathrm{K}$ avec oculaire micrométrique (fond clair, fond noir).

Les spicules sont préparés par digestion dans une solution à $30 \%$ d'hypochlorite de sodium $(2,6 \%$ de chlore actif), rinçage à l'eau, montage 
dans l'eau ou bien dans l'Aquatex ${ }^{\circledR}$. Les dimensions des spicules (20 de chaque catégorie mesurés) sont indiquées ainsi $a-b-c x d-e-f$ où $a, b$ et $c$ désignent respectivement les longueurs minimum, moyenne et maximum et $d$, e et $f$ les épaisseurs (mêmes conventions). Les coupes épaisses (environ 0,3 $\mathrm{mm}$ ) pour l'observation du squelette ont été réalisées à main levée puis montées et observées dans l'eau, dans l'Aquatex ${ }^{\circledR}$ ou, après déshydratation, dans le baume du Canada.

Les spécimens sont conservés dans l'éthanol à $95 \%$ vol.

Parmi les spécimens recueillis dans la Darse du Pacifique (port du Havre, Manche orientale), cinq spécimens ont été déposés dans la collection Vacelet, Muséum national d'histoire naturelle, Paris, sous les index MNHN-DJV177 à MNHN-DJV181. Les autres spécimens du port du Havre et les préparations microscopiques sont conservés dans les collections de la CSLN (Le Havre) et de l'association Port Vivant (Le Havre).

\section{RÉSULTATS}

\subsection{Localisation}

La population havraise d'éponges a été repérée pour la première fois lors d'une exploration à pied par l'équipe de la CSLN le 29 avril 2014 (coefficient 98) dans les enrochements de la berge nord de la darse du Pacifique, port du Havre (voir le paragraphe « Distribution et écologie »).

\subsection{Systématique}

Phylum Porifera Grant 1836

Class Demospongiae Sollas, 1885
Order Poecilosclerida Topsent, 1928 Suborder Myxillina Hajdu, van Soest \& Hooper, 1994

Family Coelosphaeridae Dendy, 1922 Genus Celtodoryx Perez, Perrin, Carteron, Vacelet \& Boury-Esnault, 2006

Celtodoryx ciocalyptoides (Burton, 1935)

Synonymie (reprise de Henkel \& Janussen, 2011)

Cornulum ciocalyptoides: Burton, 1935 : 72-73, Fig. 4 ; Koltun, 1959 : 2526, Fig. 3 ; Khodakovskaya, 2003 : 76, pl. $1 ; 2005: 210$, planche 1 .

Homoeodyctia ciocalyptoides : Koltun, 1971 : 93, Fig. 48, pl. XI(3) ; Hoshino, $1987: 26$.

Coelosphaera physa (non sensu Schmidt, 1875) : Sim \& Byeon, 1989 : 44, pl. X, Fig. 1-6.

Celtodoryx girardae: Perez et al., 2006 : 205-214, Fig. 2-3 ; van Soest et al., 2007 : 1738, Fig. 1D, 2B, 3B.

Isodyctia ciocalyptoides: van Soest 2009.

Celtodoryx ciocalyptoides: Henkel \& Janussen, 2011 : 348-351, Fig. 2 A, C-H, 3 A-O.

\section{Description}

Forme, taille, surface, couleur et consistance (Fig. 2, A-E, G)

La base de l'éponge, parfois recouverte par une pellicule sédimentaire (Fig. 2 C) varie d'une croûte épaisse à une masse très épaisse. II s'en élève des extensions irrégulières. Ces extensions, mesurant le plus souvent 0,5 à $2 \mathrm{~cm}$ de hauteur, varient considérablement en taille, forme et densité. Elles peuvent être columnaires, irrégulières et de forme déchiquetée, fréquemment 


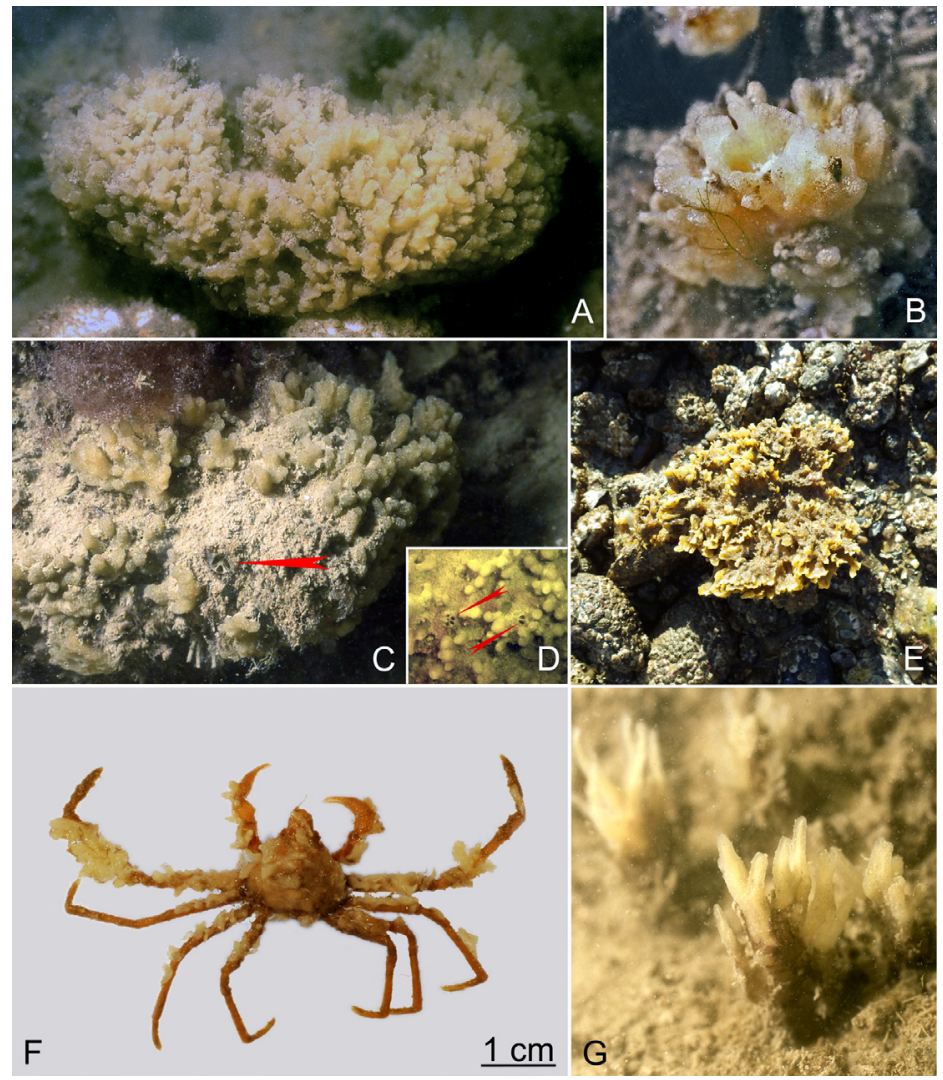

Fig. 2. Celtodoryx ciocalyptoides (Burton, 1935), Darse du Pacifique, Port du Havre, Manche orientale, 2014. Images A, D : François-Xavier Huet ; B, C, F, G : Gérard Breton ; E : Chloé Dancie. Images sousmarines sauf $E, F$.

A : Vue d'ensemble d'un spécimen. 08.06.2014. B : Détail d'une extension. 08.06.2014. C : Un spécimen avec la partie supérieure de l'éponge partiellement cachée par le sédiment. La flèche désigne un oscule. 08.06.2014. D : Deux oscules (flèches) s'ouvrant à la partie supérieure de l'éponge. 12.07.2014. E : Vue aérienne d'un spécimen émergé à basse mer parmi les blocs rocheux. 15.05.2014. F : Inachus phalangium (Fabricius, 1775) portant de nombreuses repousses de Celtodoryx ciocalyptoides sur le céphalothorax et les péréiopodes. 08.06.2014. G : Une extension d'un grand spécimen se terminant en un « buisson 》 arborescent très déchiqueté de rameaux verticaux, parallèles, serrés, interprétés ici comme une forme de croissance développée en un mois. 12.07.2014.

Fig. 2. Celtodoryx ciocalyptoides (Burton, 1935), Darse du Pacifique, Port of Le Havre, Eastern Channel, 2014. Pictures A, D: François-Xavier Huet; B, C, F, G: Gérard Breton; E: Chloé Dancie. Underwater pictures except E, F.

A: Overall view of a specimen. 08.06.2014. B: Close up of an extension. 08.06.2014. C: A specimen with the upper surface of the sponge partly hidden by the sediment. The arrow shows an oscule. 08.06.2014. D: Two oscules (arrows) opening on the upper part of the sponge. 12.07.2014. E: Field view of a specimen, emerged at low tide within blocks. 15.05.2014. F: Inachus phalangium (Fabricius, 1775) with numerous small offshoots of Celtodoryx ciocalyptoides on the cephalothorax and pereiopods. 08.06.2014. G: An extension of a large specimen ending in a very ragged dendritic "bush" of vertical, parallel, tightly packed shoots, thought here to be a growth form developed within one month. 12.07.2014. 
aplaties en lame ou en feuille, parfois avec un apex méandriforme et tronqué. Dans le détail, la partie supérieure de ces extensions porte fréquemment de nombreuses digitations (Fig. 2 B). Le 12 juillet 2014, nous notons que presque toutes les extensions des plus grands spécimens se terminent par un «buisson » dendritique de digitations parallèles et serrées d'aspect extrêmement déchiqueté (Fig. 2 G) bien différent des extensions columnaires, en lame, à apex tronqué majoritairement observées sur les mêmes spécimens le 8 juin 2014. Les oscules, peu nombreux, sont localisés sur la surface supérieure plate de l'éponge (Fig. 2C,D). Aucune forme de croissance globulaire ou en chou-fleur n'a été observée. Les plus grands spécimens couvrent approximativement $0,1 \mathrm{~m}^{2}$. La couleur est jaune pâle. La consistance est molle, non élastique, les tissus de l'éponge sont facilement déchirés. Quelques spécimens (pas tous) produisent une quantité importante de mucus lorsqu'ils sont déchirés. La membrane ectosomique des spécimens conservés en alcool est facilement séparée.

\section{Squelette}

Le squelette de l'ectosome est un assemblage tangentiel lâche de mégasclères isolés formant un réseau mal défini, au sein duquel sont dispersés de nombreux microsclères. Le squelette ectosomique incorpore du matériel étranger : particules sédimentaires, diatomées et fragments d'épibiontes (Fig. 3 F). Le squelette du choanosome, dense, plumoréticulé est constitué de faisceaux discrètement anastomosés mesurant 40 à $150 \mu \mathrm{m}$ de diamètre et formant des travées se terminant perpendiculairement à la surface par des «brosses » de mégasclères parallèles, formant parfois une palissade sous-ectosomique (Fig. 3 A).

\section{Spicules}

Les mégasclères de notre matériel sont des tylotes et des strongyles, mais des formes intermédiaires entre les deux types sont également présentes. Les tylotes sont minces, en forme de coton-tige, avec une hampe droite et cylindrique; leurs tyles portent un nombre variable mais souvent important de petites épines toutes de même taille et régulièrement espacées (Fig. $3 \mathrm{~B}, \mathrm{D}, \mathrm{G}$ ). Les strongyles, plus épais que les tylotes, sont droits ou légèrement courbes, souvent avec quelques épines apicales, peu nombreuses et irrégulières (Fig. $3 \mathrm{C}$ ). Certains sont des anisostrongyles à extrémités dissymétriques. Les vrais styles (c'est-à-dire avec une extrémité pointue) sont très rares. Bien que ces deux types de mégasclères ne soient pas strictement localisés, nous avons noté que 1) les mégasclères du choanosome sont légèrement plus longs que ceux de l'ectosome ; 2) quelques spécimens, ou quelques régions de certains spécimens, montrent une couche sous-ectosomique de vrais tylotes en coton-tige pourvus de tyles épineux qui forment les «brosses » ou les éventails distaux terminant les faisceaux ascendants de mégasclères du squelette du choanosome.

Les microsclères appartiennent à deux types. Les isochèles arqués se répartissent entre deux catégories de taille distinctes, les plus grands étant 

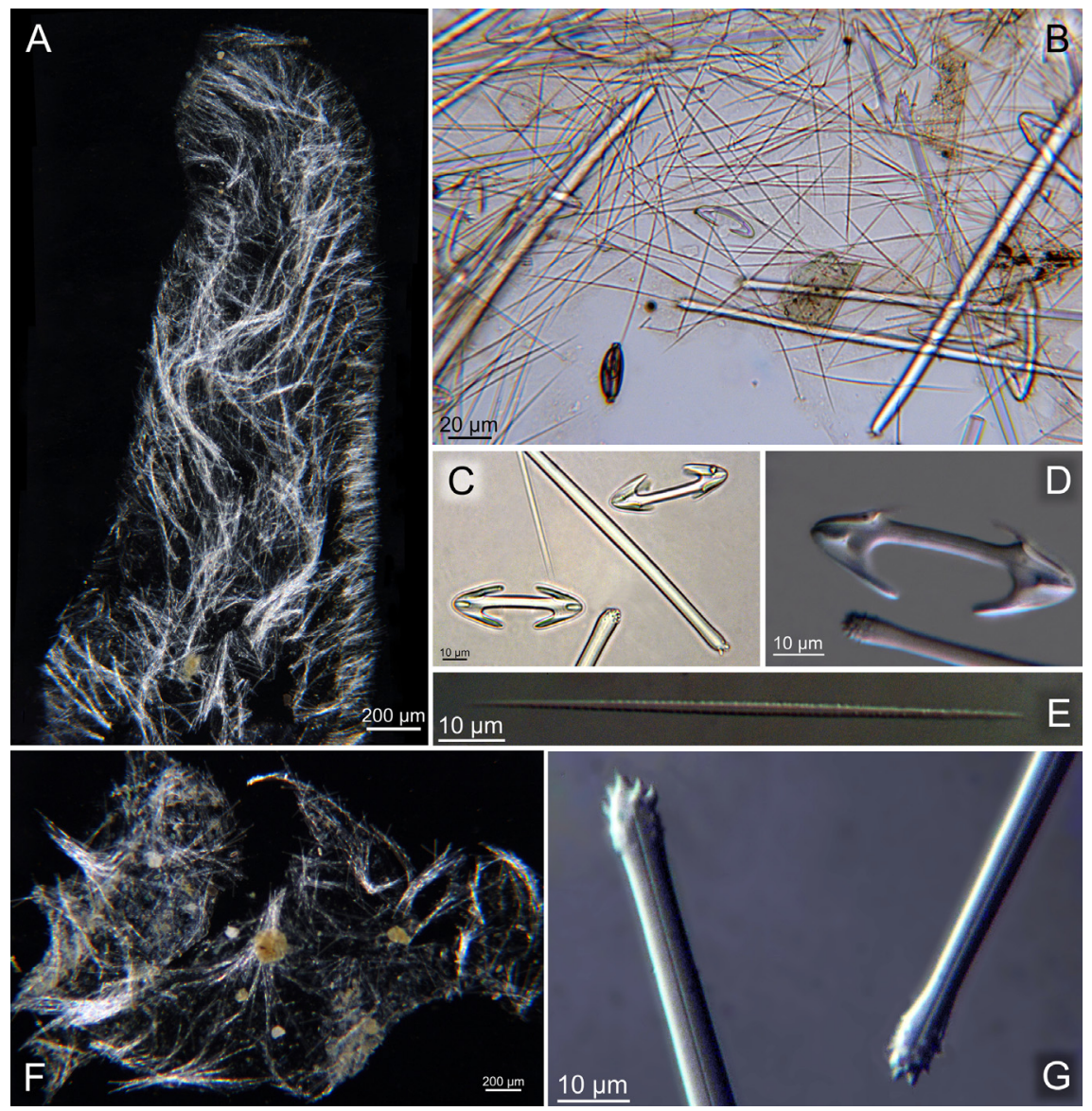

Fig. 3. Celtodoryx ciocalyptoides (Burton, 1935), Darse du Pacifique, Port du Havre, Manche orientale, 2014. Squelette et spicules. A-E, G: spécimen non numéroté, 29.04.2014 ; F : MNHN DJV 181, 12.07.2014. A, F : fond noir. B, C : fond clair. D, E, G : Contraste interférentiel. Photos : Gérard Breton. $A$ : Coupe à main levée épaisse dans une extension. Faisceaux ascendants formant un squelette plumoréticulé se terminant en brosses en éventail à la surface. B : Spicules de l'ectosome, tylotes en cotontiges, strongyles, isochèles arqués et nombreux oxychètes. Remarquer la diatomée incorporée dans le squelette de l'ectosome. C : Strongyles, isochèles arqués and oxychète. D : Tyle d'un tylote en cotontige et isochèle arqué. $\mathrm{E}:$ Oxychète. Remarquer les micro-épines visibles et le canal axial. $\mathrm{F}$ : Squelette de l'ectosome, éventails de spicules, avec du matériel étranger incorporé. G : Tyles de tylotes en cotontige. Le canal axial des spicules est visible.

Fig. 3. Celtodoryx ciocalyptoides (Burton, 1935), Darse du Pacifique, port of Le Havre, Eastern Channel, 2014. Skeleton and spicules. A-E, G: unnumbered specimen, 29.04.2014; F: MNHN DJV 181, 12.07.2014. A, F: dark field. B, C: bright field. D, E, G: DIC. Pictures: Gérard Breton.

A: Hand thick section of an extension. Ascending bundles forming a plumo-reticulate skeleton fanning out in surface brushes. B: Ectosomal spicules, "Q-tips" tylotes, strongyles, arcuate isochelae and numerous oxychaetes. Note the diatom incorporated as foreign material in the ectosomal skeleton. C: Strongyles, arcuate isochelae and oxychaete. D: Tyle of a "Q-tip" tylote and arcuate isochela. E: Oxychaete. Note the visible microspines and the axial canal. F: Ectosomal skeleton, fans of spicules, with incorporated foreign material. G: Tyles of "Q-tip" tylotes. The axial canal of the spicules is visible. 
beaucoup plus abondants que les plus petits. La forme réduite des isochèles décrite par Henkel \& Janussen (2011: 349 , Fig. $3 \mathrm{~K}$ ) est très rare dans notre matériel. Les oxychètes sont très abondants, fins, pointus aux deux extrémités. Un examen en microscopie photonique, avec un bon objectif à immersion et en contraste interférentiel selon Nomarski, permet de montrer que ces oxychètes sont micro-épineux.

\section{Dimensions des spicules}

Ces dimensions ont été mesurées sur le spécimen MNHN-DJV181, darse du Pacifique, port du Havre, mai 2014.

$\begin{array}{ll}\text { Échantillon } & \begin{array}{l}\text { Mégascleres : } \\ \text { d'ectosome seul }\end{array} \\ & \begin{array}{l}\text { 4-7,-8-11,6 } \mu \mathrm{m} . \\ \text { Mégascleres : }\end{array} \\ \text { Échantillon } & \begin{array}{l}\text { 158-250-368 } \mu \mathrm{m} \times \\ \text { de choanosome }\end{array} \\ \text { seul } & 4,8-8,3-12 \mu \mathrm{m} . \\ \text { Échantillon } & \text { Mégascleres : } \\ \text { d'ectosome + } & 180-222-372 \mu \mathrm{m} \times \\ \text { choanosome } & 4-6,3-8,8 \mu \mathrm{m} . \\ \text { Grands isochèles } & 48-54-64 \mu \mathrm{m} . \\ \text { arqués } & \\ \text { Petits isochèles } & 18-24-26 \mu \mathrm{m} . \\ \text { arqués } & \\ \text { Oxychètes } & 78-83-92 \mu \mathrm{m} .\end{array}$

\subsection{Distribution et écologie}

Toute la population est localisée sur les berges nord et nord-est de la darse du Pacifique (Port du Havre), entre $+1 \mathrm{~m}$ et $-4,8 \mathrm{~m}$ de profondeur $(0=$ Zéro des Cartes Marines = Lowest Astronomical Tides), mais elle est particulièrement dense dans les enrochements entre $+1 \mathrm{~m}$ et $-0,6 \mathrm{~m}$. Cette dernière limite inférieure des populations denses de $C$. ciocalyptoides est la limite bathymétrique où le fond sédimentaire se raccorde aux enrochements, mais des spécimens beaucoup plus dispersés croissent sur des substrats solides isolés jusqu'à-4,8 m. La limite occidentale de la distribution de cette éponge est $49^{\circ} 28,379^{\prime} \mathrm{N}$; 0 $0^{\circ} 10,254^{\prime}$ E (WGS 84). La limite orientale est $49^{\circ} 28,377^{\prime} \mathrm{N} ; 0^{\circ}$ 10,387 'E. L'éponge colonise préférentiellement la surface supérieure et les côtés obliques ou verticaux des blocs. Elle s'avère très tolérante au dépôt de matières en suspension: la surface supérieure de la base de l'éponge est souvent couverte de sédiments d'où semblent émerger les extensions (Fig. 2 C). Les oscules s'ouvrent dans des zones de la surface de l'éponge relativement protégées du dépôt de matières en suspension.

Le tableau I dresse la liste des organismes benthiques de la macrofaune et de la macroflore qui ont été reconnus au contact ou à proximité immédiate de $C$. ciocalyptoides, dans une perspective synécologique et dans le but d'offrir une comparabilité avec les études précédentes (cf. Perez et al., 2006 : 209, Tab. I).

\section{DISCUSSION}

\subsection{Taxinomie}

Tous les caractères morphologiques de l'éponge trouvée au Havre concordent avec ceux des spécimens du golfe du Morbihan (Perez et al., 
Tableau I. Liste des organismes reconnus au contact ou à proximité immédiate des Celtodoryx ciocalyptoides, darse du Pacifique, port du Havre, 2014. Dans chaque grand groupe informel, les taxons sont rangés par ordre alphabétique.

Table I. List of the organisms found on contact with, or close to, Celtodoryx ciocalyptoides, Darse du Pacifique, Le Havre port, 2014. In each informal group, taxa are in alphabetical order.

\begin{tabular}{|c|c|c|}
\hline Groupe & Taxon & Remarques \\
\hline \multicolumn{3}{|c|}{ Organismes fixés ou peu mobiles } \\
\hline Procaryotes & $\begin{array}{c}\begin{array}{c}\text { Spirulina subsalsa Oersted ex Gomont, } \\
1892\end{array} \\
\end{array}$ & $\begin{array}{l}\text { Des tapis de ce taxon croissent } \\
\text { sur le spongiaire }\end{array}$ \\
\hline \multirow[t]{6}{*}{ Algues } & $\begin{array}{c}\text { Aglaothamnion bipinnatum } \\
\text { (P.L. Crouan \& H.M. } \\
\text { Crouan) Feldmann \& Feldmann, } 1867\end{array}$ & \\
\hline & \begin{tabular}{|} 
Bryopsis plumosa (Hudson) C. Agardh, \\
1823
\end{tabular} & \\
\hline & $\begin{array}{c}\text { Ceramium cimbricum H.E. Petersen, } \\
1924\end{array}$ & \\
\hline & $\begin{array}{c}\text { Cladophora cf. laetevirens (Dillwyn) } \\
\text { Kützing, } 1843\end{array}$ & \\
\hline & Heterosiphonia japonica Yendo, 1920 & \\
\hline & Ulva sp. ou spp. (“Enteromorpha") & \\
\hline \multirow{5}{*}{$\begin{array}{c}\text { Spongiaires } \\
\text { (1) }\end{array}$} & Halichondria bowerbanki Burton, 1930 & \\
\hline & Halichondria panicea (Esper, 1794) & \\
\hline & $\begin{array}{l}\text { Hymeniacidon perlevis } \\
\text { (Montagu, 1814) }\end{array}$ & \\
\hline & Mycale macilenta (Bowerbank, 1866) & \\
\hline & Suberites ficus (Johnston, 1842) & \\
\hline \multirow[t]{3}{*}{ Cnidaires } & Obelia dichotoma (Linnaeus, 1758) & \multirow{2}{*}{$\begin{array}{l}\text { Des stolons d'Obelia sp. } \\
\text { colonisent la surface de } \\
\text { Celtodoryx ciocalyptoides }\end{array}$} \\
\hline & Obelia longissima (Pallas, 1766) & \\
\hline & $\begin{array}{l}\text { Sagartiogeton undatus } \\
\text { (Linnaeus, 1767) }\end{array}$ & $\begin{array}{l}\text { Sur un spécimen de } C \text {. } \\
\text { ciocalyptoides à }-4,8 \mathrm{~m}\end{array}$ \\
\hline \multirow[t]{6}{*}{ Polychètes } & Bispira fabricii (Krøyer, 1856) & Sensu Fauvel (1927) \\
\hline & Dipolydora coeca (Örsted, 1843) & \\
\hline & Hydroides ezoensis Okuda, 1934 & $\begin{array}{c}\text { Les tubes de ce polychète peuvent } \\
\text { être recouverts par } C \text {. } \\
\text { ciocalyptoides }\end{array}$ \\
\hline & Pholoe inornata Johnston, 1839 & \\
\hline & Syllidia armata Quatrefages, 1866 & \\
\hline & Syllis gracilis Grube, 1840 & \\
\hline
\end{tabular}


Tableau I. Suite.

Table I. Continued.

\begin{tabular}{|c|c|c|}
\hline Groupe & Taxon & Remarques \\
\hline \multicolumn{3}{|c|}{ Organismes fixés ou peu mobiles } \\
\hline \multirow[t]{4}{*}{ Mollusques } & Mytilus edulis Linnaeus, 1758 & \\
\hline & Patella vulgata Linnaeus, 1758 & \\
\hline & Littorina littorea (Linnaeus, 1758) & \\
\hline & Nassarius reticulatus (Linnaeus, 1758) & Rampe sur C. ciocalyptoides \\
\hline Crustacés & Balanus crenatus Bruguière, 1789 & $\begin{array}{c}\text { Peut être recouverte par } C \text {. } \\
\text { ciocalyptoides }\end{array}$ \\
\hline \multirow[t]{5}{*}{ Ascidies } & Ascidiella aspersa (Müller, 1776) & $\begin{array}{l}\text { Des cordons de C. ciocalyptoides } \\
\text { envahissent une grande Ascidiella } \\
\text { aspersa. Dans un stade ultérieur, } \\
\text { l'ascidie peut être complètement } \\
\text { recouverte, sauf les siphons }\end{array}$ \\
\hline & Botryllus schlosseri (Pallas, 1766) & \\
\hline & Ciona intestinalis (Linnaeus, 1767) & \\
\hline & Corella eumyota (Traustedt, 1882) & \\
\hline & Didemnum vexillum Kott, 2002 & $\begin{array}{l}\text { Se développe sur une } C \text {. } \\
\text { ciocalyptoides à }-4,8 \mathrm{~m} \text {. }\end{array}$ \\
\hline \multicolumn{3}{|c|}{ Faune vagile } \\
\hline \multirow[t]{8}{*}{ Crustacés } & Carcinus maenas (Linnaeus, 1758) & \\
\hline & Inachus phalangium (Fabricius, 1775) & $\begin{array}{c}\text { Un individu portant de nombreuses } \\
\text { repousses de } C \text {. ciocalyptoides sur le } \\
\text { céphalothorax et les péréiopodes } \\
\text { (Fig. } 2 \mathrm{~F} \text { ) }\end{array}$ \\
\hline & $\begin{array}{l}\text { Hemigrapsus sanguineus } \\
\text { (De Haan, 1835) }\end{array}$ & \\
\hline & $\begin{array}{c}\text { Hemigrapsus takanoi Asakura \& } \\
\text { Watanabe, } 2005\end{array}$ & Dont des juvéniles \\
\hline & Pagurus bernardhus (Linnaeus, 1758) & \\
\hline & Palaemon sp. & Juvéniles \\
\hline & Pisidia longicornis (Linnaeus, 1767) & $\begin{array}{l}\text { S'abrite dans les anfractuosités de } \\
\text { l'éponge }\end{array}$ \\
\hline & Porcellana platycheles (Pennant, 1777) & \\
\hline \multirow[t]{5}{*}{ Poissons } & Anguilla anguilla (Linnaeus, 1758) & \\
\hline & $\begin{array}{l}\text { Gobiusculus flavescens } \\
\text { (Fabricius, 1779) }\end{array}$ & \\
\hline & Nerophis lumbriciformis (Jenyns, 1835) & \\
\hline & Pholis gunellus (Linnaeus, 1758) & \\
\hline & Pomatoschistus sp. ou spp. & \\
\hline
\end{tabular}

(1) Haliclona cinerea (Grant, 1826) n'a pas été rencontrée à proximité de $C$. ciocalyptoides, bien qu'elle soit présente darse du Pacifique. 
2006), de l'Oosterschelde (van Soest et al., 2007) et du Pacifique nord-ouest (Henkel \& Janussen, 2011). Les différences notées sont mineures et, au plus, de niveau infraspécifique : aucune forme de croissance sphérique ou en chou-fleur, taille maximale de l'éponge plus faible, extensions jamais fistuleuses. Nous avons noté que presque tous les plus grands spécimens portent des extensions prolongées par un « buisson » dendritique d'aspect déchiqueté un mois après avoir porté des extensions columnaires, en lames ou à aspect tronqué. Cette morphologie particulière des apex des extensions pourrait donc constituer une forme de croissance originale (Fig. $2 \mathrm{G}$ ). Les mesures des dimensions des spicules semblent un peu plus dispersées que celles publiées pour les autres populations européennes ou pacifiques. Ces différences notées sont probablement significatives d'une population jeune et d'une diversité génétique limitée.

Sur la base des caractères morphologiques et anatomiques, nous suggérons que les spécimens observés dans le port du Havre appartiennent à la même espèce que ceux décrits de Bretagne, des Pays-Bas et du Pacifique nord-ouest.

\section{2 Écologie}

C. ciocalyptoides est implantée dans le port du Havre sur une surface limitée, dans un bassin de marée, mais à $6,5 \mathrm{~km}$ de l'entrée du port. Breton (2005) a montré que la zonation du domaine paralique (sensu Guélorget et Perthuisot, 1983) peut être appliquée au port du Havre. Dans cette optique, la population de $C$. ciocalyptoides s'est développé en zone III de la zonation de Guélorget \& Perthuisot (1983), ce que confirme la liste des organismes associés (Tab. I). II est remarquable que les populations asiatiques se soient également développées dans des environnements semi-fermés (Henkel \& Janussen, 2011) donc paraliques, ainsi que celles de Bretagne ou des Pays-Bas. Par contre, les populations de Bretagne méridionale et d'Asie se développent dans des zones à hydrodynamisme fort, les courants étant gouvernés par une marée semi-diurne et sont donc rhéophiles. Au contraire, les courants de marée dans la darse du Pacifique (port du Havre) sont très faibles: la population havraise de C. ciocalyptoides n'est pas rhéophile.

La température dans le port du Havre (moyennes mensuelles de 6 à $21^{\circ} \mathrm{C}$ pendant la dernière décennie) est proche de la température des localités pacifiques citées par Henkel \& Janussen (2011) : 4-25 ${ }^{\circ} \mathrm{C}$. La population havraise tolère une brève émersion à basse mer en vive-eau, puisqu'elles se trouvent jusqu'à $+1 \mathrm{~m}$. C'est une différence écologique notable avec les autres populations.

C. ciocalyptoides se trouve-t-elle en mer ouverte (=en domaine thalassique sensu Guélorget \& Perthuisot, 1983) en Europe ? La littérature ne mentionne à notre connaissance aucune occurrence, ce que confirme van Soest (2015). Peut-être l'espèce est-elle présente mais discrète et n'a pas encore été repérée. Sichel \& Bunel (2012 : pl. X, Fig. 53) montrent une photo convaincante de cette espèce [49 $25^{\circ} 04^{\prime \prime} \mathrm{N}$; $00^{\circ} 17^{\prime} 98^{\prime \prime}$ W (WGS 84) ; $z=-24 \mathrm{~m}$ ]. Mais sans examen des spicules, cette 
possible occurrence en mer ouverte reste discutable. C. ciocalyptoides est donc opportuniste, suspensivore et paralique. Elle partage ces caractéristiques avec quelques-unes des autres espèces récemment introduites enregistrées dans le port du Havre et dont la liste est donnée par Breton (2014) : Ficopomatus enigmaticus (Fauvel, 1923), Mnemiopsis leidyi (A. Agassiz, 1860), Hydroides ezoensis Okuda, 1934, Tricellaria inopinata d'Hondt \& Occhipinti Ambrogi, 1925, Botrylloides spp., Didemnum vexillum Kott, 2002. Toutes ont connu d'importantes fluctuations démographiques dans le port du Havre où certaines sont considérées comme invasives (Breton, 2014, 2015).

\subsection{Date et mode d'introduction dans le port du Havre}

Depuis le début des années 80, les biologistes du Muséum d'histoire naturelle du Havre, avec l'aide d'une équipe de biologistes et plongeurs volontaires, ont inventorié les populations du port du Havre et étudié leur évolution (Breton, 1981; Breton et al., 1996). Breton (2005) a synthétisé les résultats de 25 années de surveillance en donnant une liste des taxons présents dans le port du Havre. Depuis 2006, ce programme est piloté par Port Vivant, une association de sciences participatives, et Breton (2014) a actualisé et complété les données précédentes et commenté sur les espèces allochtones et invasives dans le port du Havre. De plus, la Cellule de Suivi du Littoral Normand poursuit depuis plusieurs décennies un suivi de l'endofaune du port du Havre, comprenant de nombreuses observations incidentes de l'épifaune. Le site d'implantation de $C$. ciocalyptoides avait été exploré au moins une fois par an par Port Vivant depuis 2010. Nous pouvons donc conclure que $C$. ciocalyptoides est un nouvel arrivant dans le port du Havre et non pas une espèce non repérée issue d'une introduction plus ancienne. La date d'introduction est donc 2013 ou 2014.

Henkel \& Janussen (2011), après van Soest et al. (2007) et Perez et al. (2006) invoquent l'ostréiculture de l'huître pacifique Crassostrea gigas (Thunberg, 1793) comme possible vecteur d'introduction de C. ciocalyptoides du Pacifique nord-ouest dans l'Atlantique nord-est. Ce vecteur, plausible pour les populations de Bretagne méridionale et des Pays-Bas, ne peut pas être invoqué pour expliquer l'introduction dans le port du Havre. En effet, l'établissement ostréicole le plus proche est situé à Courseulles-sur-mer (Calvados), et il utilise des cuves étanches dans l'estuaire de la Seulles. Il est situé à $52 \mathrm{~km}$ de la darse du Pacifique (Port du Havre). La population de C. ciocalyptoides s'est développée dans le port du Havre à $0,4 \mathrm{~km}$ d'une écluse importante, l'écluse François $\mathrm{l}^{\mathrm{er}}$, empruntée par de nombreux navires venant du Pacifique nord-ouest, ou, en plus faible nombre, des Pays-Bas. D'importants terminaux accueillant des porte-conteneurs de mêmes provenances sont situés de 0,6 à $1,5 \mathrm{~km}$ de la population d'éponges. Le vecteur le plus plausible d'introduction de $C$. ciocalyptoides dans le port du Havre est le trafic maritime, le biofouling sur la coque d'un navire. 


\section{CONCLUSION}

Sauleau \& Izard (2012) se demandent si $C$. ciocalyptoides est un véritable compétiteur invasif ou un compétiteur « normal » et ils proposent un suivi de ses aptitudes démographiques et de ses relations biotiques dans la ria d'Etel. Nous pouvons nous poser la même question à propos des populations havraises.

La liste des taxons associés à $C$. ciocalyptoides, très semblable à celle qui serait dressée pour le même plan d'eau en dehors de la présence de l'éponge montre qu'elle n'élimine aucun autre taxon et sert même d'abri à certaines espèces (e.g. Pisidia longicornis). Breton (2014) a montré que la plupart des espèces animales invasives d'introduction récente dans le port du Havre avaient connu une explosion démographique initiale importante suivie d'une décroissance, puis d'oscillations annuelles ou pluri-annuelles.

Nous ne pouvons pas prédire si $C$. ciocalyptoides sera un compétiteur dans le port du Havre avec d'autres organismes paraliques suspensivores ou non. Une évolution possible est qu'à l'instar des populations néerlandaise et bretonne, elle devienne invasive au moins temporairement au sein de nos bassins paraliques mais reste discrète ou absente dans la mer ouverte proche.

Seul le suivi de la population de la darse du Pacifique du port du Havre au cours des prochaines années permettra de préciser quel schéma s'applique.

\section{REMERCIEMENTS}

Nous remercions les autorités du Grand Port Maritime du Havre (GPMH) pour les autorisations de plonger et de prospecter à pied, les responsables du Département Environnement du GPMH pour l'intérêt constant qu'ils ont manifesté pour nos recherches, Jean Vacelet, Rob van Soest et Daniela Henkel pour l'envoi de documentation. Nos remerciements s'adressent également, pour leur aide sur le terrain, aux plongeurs de l'association Port Vivant et à Alain Corthésy, ainsi qu'à Séverine Dubut et Bastien Chouquet.

\section{RÉFÉRENCES BIBLIOGRAPHIQUES}

Breton G., 1981. Observation sur l'écologie et les peuplements des bassins du port du Havre (France). Bulletin trimestriel de la Société géologique de Normandie et des Amis du Muséum du Havre 68 (4) : 45-58.

Breton G., 2005. Le port du Havre (Manche Orientale, France) et ses peuplements : un exemple de domaine paralique en climat tempéré. Bulletin de la Société Zoologique de France 130 (4) : 382- 423.

Breton G., 2014. Espèces introduites ou invasives des ports du Havre, d'Antifer et de Rouen (Normandie, France). Hydroécologie Appliquée 18 : 23-65.

Breton G., Girard A. \& Lagardère J.-P., 1996. Espèces animales benthiques des bassins du port du Havre (Normandie, 
France) rares, peu connues ou nouvelles pour la région. Bulletin trimestriel de la Société géologique de Normandie et des Amis du Muséum du Havre 82 (3) : 7-28.

Burton M., 1935. Some sponges from the Okhotsk Sea and the Sea of Japan. Exploration des Mers de l'URSS22 :6179.

Fauvel P., 1927. Polychètes sédentaires. Faune de France, Volume 16, Paul Lechevalier, Paris : $494 \mathrm{p}$.

Geller J.B., 1999. Decline of a native mussel masked by sibling species invasion. Conservation Biology 13 (3) : 661-664.

Guélorget O. \& Perthuisot J.-P., 1983. Le domaine paralique : expressions géologiques, biologiques et économiques du confinement. Presses de l'École Normale Supérieure, Paris Travaux du Laboratoire de Géologie 16 : 136 p.

Henkel D. \& Janussen D., 2011. Redescription and new records of Celtodoryx ciocalyptoides (Demospongiae: Poecilosclerida) - a sponge invader in the north east Atlantic Ocean of Asian origin? Journal of the Marine Biological Association of the United Kingdom 91 (2) : 347355.

Jousson O., Pawlowski J., Zaninetti L., Zechman E.W., Dini F., Di Guiseppe G., Woodfield R., Millar A. \& Meinesz A., 2000. Invasive alga reaches California. Nature 408 : 157-158.

Kuris A. M. \& Culver C.S., 1999. An introduced sabellid polychaete pest infesting cultured abalones and its potential spread to other California gastropods. Invertebrate Biology 118 : 391-403.
Occhipinti-Ambrogi A., 2007. Global change and marine communities: alien species and climate change. Marine Pollution Bulletin 55 : 342-352

Perez Th., Perrin B., Carteron S., Vacelet J. \& Boury-Esnault N., 2006. Celtodoryx girardae, gen. nov. sp. nov., a new sponge species (Poecilosclerida: Demospongiae) invading the Gulf of Morbihan (North East Atlantic, France). Cahiers de Biologie Marine 47 : 205-214.

Sauleau P. \& Izard C., 2012. Suivi de l'éponge marine Celtodoryx ciocalyptoides dans le site Natura 2000 ria d'Etel (Morbihan). Lettre d'information des gestionnaires d'AMP de la façade atlantique, 3 (5) [disponible en ligne : http:// www.maia-network.org/accueil/ les_documents/512729/lalettredinformationdesgestionnairesdampdelafacad eatlantiqueenfrancen 3 , accessed on 12/07/2014].

Sichel F. \& Bunel N., 2012. Plongée bio du 20/11/2011. Compte rendu [disponible en ligne : http://caenplongee.org/wpcontent/uploads/CR-barge-grue-20-112011-v2.pdf accessed on 12/07/2014].

Van Soest R., 2015. Celtodoryx ciocalyptoides (Burton, 1935). World Porifera database disponible en ligne http:// www.marinespecies.org/porifera/porifera.php? $p=$ taxdetails \& $i d=559274$ accessed on 2015-05-11.

Van Soest R.W.M., de Kluijver M.J., van Bragt P.H., Faasse M., Nijland R., Beglinger E.J., de Weerdt W.H. \& de Voogd N.J., 2007. Sponge invaders in Dutch coastal waters. Journal of the Marine Biological Association of the United Kingdom 87 : 1733-1748. 